\title{
Incidencia de inteligencia creativa del docente en su práctica pedagógica de docentes durante la pandemia
}

\section{Incidence of teachers' creative intelligence in their pedagogical practice during the pandemic}

\author{
Leidy Carolina Tunjo-Guerrero ${ }^{1}$ y Yangali Vicente Judith Soledad ${ }^{2 a}$ \\ Universidad Norbert Wiener, Lima, Perú ${ }^{12}$ \\ ORCID ID: https://orcid.org/0000-0002-3710-6971 \\ ORCID ID: https://orcid.org/0000-0003-0302-5839
}

Recibido: 16 de enero de 2021

Aceptado:28 de marzo de 2021

\section{Resumen}

Este artículo muestra la incidencia de la inteligencia creativa del docente en su práctica pedagógica, a partir del estudio de 8 casos, en diferentes sectores educativos de la educación básica y media de la ciudad de Bogotá-Colombia. Para el proceso de investigación se utilizó un enfoque cualitativo correspondiente al método de fenomenología, con un diseño centrado en estudio de casos. Lo anterior produjo la efectividad de la investigación, siendo verificable la influencia de la creatividad dentro de las subcategorías "saber hacer”, "saber ser" y "saber conocer" de la práctica pedagógica desarrollada durante la pandemia del 2020. Se concluye que el proceso de la práctica pedagógica aplicada durante la situación extrema del año 2020, necesitaba características flexibles e innovadoras por parte del profesor, que resolvieran las diferentes necesidades del conocimiento de la praxis.

Palabras clave: adaptación, creatividad, pandemia, pedagogía, práctica

\section{Abstract}

This article shows the incidence of the creative intelligence of teachers in their pedagogical practice, based on the study of 8 cases, in different educational sectors of basic and secondary education in the city of Bogotá-Colombia. The research process used a qualitative approach corresponding to the phenomenology method, with a design centered on case studies. This produced the effectiveness of the research, being verifiable the influence of creativity within the subcategories "knowing how to do", "knowing how to be" and "knowing how to know" of the pedagogical practice developed during the pandemic of 2020. It is concluded that the process of the pedagogical practice applied during the extreme situation of the year 2020, needed flexible and innovative

${ }^{\mathrm{a} C}$ Correspondencia al autor:

E-mail: judith.yangali@ uwiener.edu.pe 
characteristics on the part of the teacher, which solved the different needs of the knowledge of the praxis.

Keyword: adaptation, creativity, pandemic, pedagogy, practice.

\section{Introducción}

Iniciando la pandemia por Covid-19, los docentes se enfrentaron a diferentes estados emocionales, y con ello creció la necesidad de implantar nuevas herramientas en la práctica pedagógica. A su vez, se podía identificar perceptivamente, que la educacion cambió algunos aspectos, tales como la forma de interacción dentro del ambiente de clase, hasta la cultura de cuidado séptico, convirtiendo la dinámica pedagógica del año 2020 en una metodología de enseñanza y aprendizaje adaptada a la necesidad del momento (Gonzalez, 2020; Markovits, \& Douglas, 2020; Gustafsson \& Nuga, 2020; Hacımustafaoğlu, 2020).

Por ende, los maestros implementaron estrategias en una situación inesperada, para la cual diferentes habilidades creativas, fueron evidentes. Todo lo anterior motivó el estudio en la región de Bogotá-Colombia, en una muestra de seis estudios de caso, enfocados en docentes de diferentes colegios, repartidos entre el sector oficial, privado y convenio, con la finalidad de evitar sesgos en términos de recursos educativos, entendiéndose que el nivel económico de la población educativa cambia según el sector y ubicación geográfica del colegio, y que este influye directamente a los recursos de una práctica pedagógica (Miranda, 2020; Amed \& Ahmad, 2019; Eden, 2020).

En efecto a lo anterior, la investigación enfatiza, en el estudio de casos, analizando la creatividad como una característica trial, la cual se comporta como inteligencia, habilidad o capacidad. Por su lado, la creatividad es definida por Torrance, como un proceso metacognitivo de reorganización de conocimiento en el cuál se forman métodos, con la finalidad de formular, modificar o comprobar resultados, de una forma novedosa, indicando que esta característica es inherente a la práctica dentro del ambiente pedagógico. De su definición sobresale que a nivel personal requiere diferentes técnicas personales que merecen ser analizadas. Esta característica es necesaria para el rol personal y laboral de las personas, porque construye un ideal de equilibrio y responde a la construcción novedosa de diferentes técnicas de ejecución en el ambiente de trabajo, haciendo contraste con las necesidades visibles para el saber ser, saber conocer y saber hacer del docente, siendo de esta forma una respuesta a un estímulo externo y a su vez, 
constructora de vivencias que posibilitan ser observadas, analizadas y comparadas por medio de un estudio de casos (Kotsuo et al, 2019; Extremera, 2018; Mattingly \& Kurt, 2019; Dacre et ál, 2019; Wajdi et ál, 2018; Vesely-Maillefer \& Saklofske, 2018; Harris \& Bruin, 2018; Hung \& Sitthiworachart, 2020; Haertel \& Terkowsky; 2021).

Por todo lo expuesto anteriormente, el diseño adecuado de investigación describe desde un enfoque fenomenológico la creatividad del docente, dándole importancia a la necesidad de innovar en la práctica educativa. Esta habilidad se describe dentro del contexto vivencial del docente durante la contingencia del año 2020 (Henao et al, 2019; Henao \& Marín, 2019). En suma, La pandemia no solo mostró un reto para los docentes, sino que adicionalmente, afrontaron el desafío de ser guías en sus asignaturas de una forma creativa, a la vez que tenían que encontrar la estabilidad en su vida en un momento donde la vulnerabilidad se hizo noticia común en la mayoría de ciudades del mundo, mostrando conmoción entre las diferentes comunidades educativas (Markovits \& Douglas, 2020; Eden, 2020; Mays, 2020).

Por consiguiente, la investigación pretende desde un aspecto científico y cualitativo dar el peso teórico y descriptivo a la creatividad, enfocada en la influencia sobre la praxis pedagógica ofrecida por docentes que afrontaron el desafío durante la pandemia, y adicionalmente describir hábitos y aspectos vivenciales que se convirtieron en experiencias de vida, a través de un punto psicológico y educativo, tomando como premisa el posible cambio que la educación obtuvo ante la reacción de una pandemia. Por todo lo anterior, se hizo necesaria una investigación que sirviera para un futuro en caso de volverse a presentar una situación que involucre un riesgo biológico (Gonzalez, 2020).

Por ultimo cabe resaltar que la investigación se trabajó bajo el objetivo de analizar el fenómeno respectivo a la manifestación de capacidades creativas. Y a partir de este punto, se direccionaron los objetivos específicos en describir y analizar hábitos que fortalecieran y dieran renombre a esta habilidad desapercibida y figurar su incidencia en la práctica pedagógica desarrollada durante la pandemia del año 2020.

\section{Fundamentación teórica}

\section{Creatividad}

Según Esquivias (2008; citado por Agudelo, 2019), la creatividad es un juicio donde se ocasiona un proceso metacognitivo de reorganización de conocimiento, construyendo una idea novedosa, mostrando las capacidades del ser humano. Se puede 
decir que la creatividad no solo es responsabilidad de los educadores o psicólogos, sino que diferentes disciplinas han plasmado su interés y desarrollo en la evolución de su estudio. Por otro lado, es válido afirmar que cada persona es diferente en el aspecto creativo, porque esta, tiene enlace con la personalidad, generando una tendencia que se mezcla con las preferencias personales.

Lo anterior indica que cada persona lleva un proceso diferente desarrollando conexiones distintas al realizar los procedimientos de respuesta, según experiencias y conocimientos, es observable que cada persona se sitúa en uno o varios de los estilos de la creatividad, y de esto depende que la persona explote o desarrolle su habilidad creativa. Según Garcés, 2019, los descriptores de creatividad se dividen en: estructurada, no lineal, provocada y Eureka. Cada uno de los estilos se sitúan es descriptores propios de la personalidad, haciendo hincapié en el desarrollo personal de cada persona, al contrario de Flores (2019), que muestra todo un proceso para alcanzar un nivel creativo.

Por otra parte, Garcés (2019), habla de la creatividad como una estrategia innovadora de enseñanza y por esta razón la muestra como una necesidad dentro del aula. La creatividad también depende del entorno en el que se forme el individuo, si un individuo se forma en un cambien musical, probablemente tenga la tendencia se desarrollar la creatividad con este tipo de inteligencia. Por otra parte, Rodríguez en su manual de creatividad (1995; citado por Flores, 2019), resalta que el proceso creativo tiene seis etapas, divididas en: cuestionamiento (interés), acopio de datos (observación), incubación (asimilación), iluminación (inspiración), elaboración (técnica) y comunicación (elaboración final).

\section{Práctica pedagógica}

Según el artículo 119 de Ley general de Educación, de la Ley 115 de 1994, establece que la práctica pedagógica es desarrollada por el docente en forma teórica y práctica, como parte de la praxis y del saber especifico, prestando un servicio de calidad a los protagonistas del proceso aprendizaje-enseñanza. Según lo anterior en el ambiente didáctico se hace el enlace del desarrollo conceptual, investigativo y experimental del proceso, donde se promueve el progreso de competencias y deberes básicos de aprendizaje. Adicionalmente forma un escenario intelectual entre sujetos y saberes, generando reflexión, pensamiento crítico y confrontación que valora el reconocimiento y formación disciplinar del desarrollo de sistema teórico y de aplicación (Ley General de Educación, 1994). 
Según Martínez (2017) la práctica pedagógica, se configura desde la experiencia, el cual otorga conocimientos que le dan forma las habilidades en el proceso de enseñanzaaprendizaje. En este sentido el maestro es una persona que decisiones propias, que está inmerso en un proceso metacognitivo de autoconocimiento y destrezas personales, por lo anterior debe ser capaz de gestionar sus habilidades de interacción, enseñanza y aprendizaje. Al respecto, Atlet (citado por Martinez, 2017), habla de que el docente tiene la obligación de realizar flexibilizaciones que favorezcan al estudiante, y al mismo tiempo lo vincule en un pensamiento crítico de apropiación de contenidos. Por otra parte, es importante para la praxis, el cambio de paradigma, el cual es una adaptación a las necesidades sociales con la finalidad de la construcción de nuevos saberes y la elaboración de una práctica más consiente.

Duque \& Duque (2019) definen la práctica pedagógica como el resultado de las experiencias, que edifican el ejercicio de la reflexión permanente en conceptualización y ejecución del proceso de enseñanza y aprendizaje. El espacio didáctico generado, aborda la investigación, conceptualización y experimentación de saberes, dentro de la disciplina en la que es requerida. La estrategia didáctica del docente debe estar ligada a la postura activa que necesita el estudiante, para generar los objetivos necesarios para el compromiso formado por el interés de aprender.

\section{Metodología}

Según Arispe et al (2020) es una investigación de enfoque cualitativo, que responde al método inductivo y desarrolla la fenomenología. La investigación busca consolidar la importancia y necesidad de ciertas capacidades en los docentes, para mantener el equilibrio emocional y el interés en adaptarse al cambio y generar soluciones a cierta ausencia de recursos, tiempo o espacio. Pretende profundizar en los conceptos de las capacidades socioemocionales y creativas del profesor, para construir un saber desde la parte científica, que la investigación básica trae.

La investigación es de carácter fenomenológico y el diseño es de estudio de casos. Esto por la razón de describir un fenómeno de una forma profunda, mostrando los diferentes comportamientos, desde el estudio de 6 casos distintos. Esta combinación de diseños ha mostrado grandes resultados en el ámbito de la educación, al lograr estudios a profundidad, utilizándolos como complementos el uno del otro. Desde esta forma juegan un papel importante en el procesos de estudio viendo los objetivos de análisis, desde una perspectiva integral (Brocki \& Wearden, 2006). 
Como anteriormente se describe, se utilizó el diseño de estudio de casos, expuesto por Déborah y García, (2018), como uno de los más factibles para analizar fenómenos presentados en el ámbito educativo. Adicionalmente Grinnell, Williams y Unrau (2009), y Hernández-Sampieri y Mendoza, (2008), citados por Hernández y Mendoza. (2014a), los cuales describen el estudio de casos como el diseño que requiere una disertación intensiva mediante datos provenientes de descripciones e información extensiva. Siendo así, se define "caso”, como la unidad estudiada que emite características propias dando resultado a un análisis. Un caso puede ser una persona, un grupo de personas, un fenómeno, un proceso o un evento.

En efecto, se analizó el fenómeno que constituido por la creatividad del docente, en su práctica pedagógica, durante una situación límite como es la presentada por una pandemia. Y se encontró que el diseño más efectivo era el estudio de casos, porque según los planteamientos de Green (2011), Robson (2011), Mertler y Charles (2010), así como Armenian (citado por Hernández y Mendoza, 2014b), este diseño permite describir fenómenos, identificar sus patrones en la situación planteada, provee datos para analizar ambientes, valida y produce conocimiento. Adicionalmente según Thomas (2010), el diseño por casos examina a los sujetos de forma holística, dando una mirada más global a la situación problémica. Por último, el diseño empleado es acertado para construir conocimiento y resaltar la creatividad en la práctica pedagógica, por esta razón se empleó un estudio de caso colectivo, el cual buscó construir la base teórica y sumar hallazgos al aspecto educativo desde varios puntos de vista (Hernández y Mendoza, 2014b).

El ambiente de estudio se enfocó en 6 casos de Bogotá, tomando 2 casos de convenio, 3 de institución privada y 2 de colegios oficiales, todos ellos con el factor común de ejercer una práctica pedagógica durante la pandemia por COVID-19. Lo cual presentó a la educación en una atmosfera educativa totalmente nueva, para una sociedad que está en la cuarta revolución tecnológica (Perasso, 2016). El escenario escogido muestra la praxis de forma no presencial, en colegios que llevaban decenios impartiendo clases magistrales y desarrollando estrategias de esta forma. Adicionalmente de la educación virtual, la comunidad daba respuestas durante el aislamiento preventivo en el lugar de análisis.

Por otra parte, según Hernández y Mendoza, (2014), la muestra ideal para un estudio de casos es de cinco a diez sujetos. Por esta razón se toma una muestra de seis docentes, provenientes de seis colegios distintos, ubicados en diferentes localidades de Bogotá. Se eligió una muestra de alta variedad haciéndola no probabilística intencional 
y de máxima variación, porque buscó mostrar la complejidad del fenómeno, incluyendo coincidencias o diferencias entre los seis casos (Ragin, 2013, Saumure y Given, 2008a y Palys, 2008, citado por Hernández y Mendoza, 2014). Se hizo exclusión de docentes de institutos porque no alcanzan las 30 horas laborales semanales mínimas, y docentes de primera infancia por tener parámetros de enseñanza diferentes a primaria y bachillerato.

\section{Tabla 1}

Participantes analizados

\begin{tabular}{|c|c|c|c|c|}
\hline Participante & $\begin{array}{r}\text { Estrato social de la } \\
\text { población donde labora }\end{array}$ & Asignatura & Sector & Nivel \\
\hline P1 & $2,3,4$ & Dibujo. & Privado & bachillerato \\
\hline P2 & $0,1,2$ & $\begin{array}{l}\text { Matemáticas y } \\
\text { física. }\end{array}$ & Oficial & $\begin{array}{l}\text { Primaria- } \\
\text { bachillerato }\end{array}$ \\
\hline P3 & $0,1,2$, & Sociales y filosofía. & Oficial & primaria \\
\hline P4 & $2,3,4$ & Ed. Física. & Privado & $\begin{array}{r}\text { Primaria } \\
\text { bachillerato }\end{array}$ \\
\hline P5 & 2,3 & Danzas. & Privado & \\
\hline P6 & $2,3,4$ & Ciencias naturales. & Convenio & $\begin{array}{l}\text { Bachillerato- } \\
\text { primaria }\end{array}$ \\
\hline P7 & $1,2,3$ & Ingles. & Convenio & primaria \\
\hline P8 & 3,4 & Español e inglés. & Oficial & bachillerato \\
\hline
\end{tabular}

Fuente: Elaboración propia de las investigadoras

La recolección de datos, los resultados aparecen paulatinamente, los cuales se orientan a la comprensión de experiencias, pensamientos o sucesos, principalmente analizados por observación (Hernández y Mendoza, 2014a). En suma, se trabajaron las siguientes técnicas:

\section{Tabla 2}

Técnicas e instrumentos utilizados

\begin{tabular}{cc}
\hline TÉCNICA & INSTRUMENTO \\
\hline Test psicométrico & Test CREA \\
Observación & Lista de chequeo \\
Encuesta & Instrumento \# 1 \\
Entrevista & Entrevista a profundidad \\
\hline Fuente: Elaboración propia de las investigadoras
\end{tabular}

\section{Instrumento $N^{\circ} 1$}

Se realiza la adaptación de cuatro instrumentos diferentes que miden características en la praxis pedagógica del docente desde diferentes perspectivas. La adaptación se llevó a cabo, porque no se encontró ningún instrumento que hablara de la 
praxis pedagógica en una situación límite, por esta razón se decisión obtener los ítems más relevantes para la investigación. Cuenta con 20 preguntas direccionadas a las dimensiones descritas en la matriz de trabajo. Las respuestas son para observación de las prácticas que se están aplicando.

\section{Entrevista a profundidad}

Se realizará una entrevista de máximo 15 minutos, donde se conocerán aspectos de hábitos del docente, que den indicio a su nivel de creatividad y capacidad socioemocional. Adicionalmente para conocer su pensamiento sobre la praxis pedagógica. La entrevista es utilizada como una técnica tradicional, con la finalidad de obtener información de pensamientos o historias de vida de las personas. Se debe utilizar un orden de preguntas, para no perder el hilo del objetivo. Cuando la entrevista es a profundidad, intenta crear un ambiente de confianza, donde el entrevistado se sienta segura de dar a conocer parte de su pensamiento o vida. Genera contextualización de las temáticas y a porta, flexibilidad a la obtención de datos porque incluye preguntas cerradas y abiertas. En este trabajo, la entrevista fue dirigida a escudriñar, el pensamiento de los docentes respecto a sus gustos, para establecer hábitos que fomenten la creatividad y capacidad socioemocional del docente, y adicionalmente, poder definir el cambio de la práctica pedagógica antes y durante la pandemia (Bernal, 2010; Hernández y Mendoza, 2014 ${ }^{\mathrm{a}}$, Arispe, et al., 2020).

\section{Test CREA}

Se utiliza para medir la variable de inteligencia creativa. Nació con la necesidad de vincular la creatividad con una habilidad, capacidad o inteligencia. Sus resultados cuantitativos, resultan en una traducción cualitativa entre alta, media y baja, facilitando las investigaciones de esta índole. Para la evaluación psicológica de rasgos, se utiliza factores tales como la flexibilidad, originalidad y producción. Según el resultado que obtenga la persona en el test, se puede resaltar lo siguiente:

-Creatividad alta: tienen dominio de emociones y de comportamiento, estando solos o en compañía, presenta alta actitud de interrogación.

-Creatividad media: presenta un moderado nivel de creación, tiene aplicación de estrategias de pensamiento convergente. 
-Creatividad baja: son favorecidos por un ámbito bastante permisivo, generalmente presentan bajo autocontrol o regulación de espacio y sentimientos

Se aplicó Atlas TI, a fin de analizar constructos obtenidos, categorías de código y redes entre datos. Se utilizó para el análisis de las entrevistas, instrumentos 1 , y test psicométricos, para encontrar conexiones directas e indirectas con la inteligencia creativa en el aspecto educativo y personal. Este programa facilitó las triangulaciones entre los tres sectores educativos a analizar y creó arboles de conexión los cuales fueron fructíferos para describir el fenómeno creativo (Rico, 2017).

\section{Resultados}

Los resultados obtenidos a través del estudio de 8 casos en diferentes sectores de Bogotá, describiendo las categorías: creatividad y practica pedagógica.

Tabla 3

Resultados test CREA e instrumento uno

\begin{tabular}{ccccccccc}
\hline PARTICIPANTE & P1 & P2 & P3 & P4 & P5 & P6 & P7 & P8 \\
\hline TEST CREA & Medio & Alto & Alto & Alto & Medio & Alto & Alto & Alto \\
$\begin{array}{c}\text { Adaptación } \\
\text { de guías, } \\
\text { power } \\
\text { point }\end{array}$ & $\begin{array}{c}\text { Adaptación } \\
\text { de } \\
\text { materiales } \\
\text { reutilizables }\end{array}$ & Historietas & $\begin{array}{c}\text { Juegos } \\
\text { de star } \\
\text { Wars }\end{array}$ & $\begin{array}{c}\text { Creación de } \\
\text { coreografías } \\
\text { familiares }\end{array}$ & $\begin{array}{c}\text { Huertas } \\
\text { caseras }\end{array}$ & $\begin{array}{c}\text { Juego } \\
\text { de } \\
\text { roles }\end{array}$ & $\begin{array}{c}\text { Adaptación } \\
\text { de comics }\end{array}$ \\
\hline
\end{tabular}

Fuente: Elaboración propia

\section{Creatividad}

Según la tabla 3, los 8 integrantes de la muestra revelaron resultados de inteligencia creativa entre el rango medio y alto, evidenciando respuestas y adaptaciones diferentes. Los casos 1, 2 y 3 se destacaron por construir ideas según sus conocimientos o percepciones de adaptación que consideraban (Snyder et al., 2019; Blanquiz \& Villalobos, 2018; Østbye, 2018). Y en efecto, según las entrevistas se evidenció la adaptación de materiales que encontraban en el hogar, todas ellas con la finalidad de utilizar recursos que estuviesen al alcance y no generara algún costo. Lo anterior demuestra el dominio de una producción innovadora orientada a generar una nueva idea respecto a un objeto, como lo indica Mumford et al. (2018). La recursividad del docente, fue dominada por una necesidad generada durante la pandemia, acreditando una relación entre su inteligencia creativa y su desarrollo organizacional, que según Cheung et al. 
(2020) es producto de compromisos implícitos en un líder, el cual es capaz de guiar un grupo con hallazgos en su presente inmediato, generando una respuesta positiva y motivadora para aquellos que lo escuchan. Lo anterior, también enfatiza en una percepción de claridad respecto a una temática (Elisondro, 2018).

Por otra parte, según las entrevistas, aquellos que obtuvieron mejores resultados en su test de inteligencia creativa, conceptualizaron voluntariamente la creatividad como un pensamiento del diario vivir, conectado el respaldo de su habilidad creativa con las tareas cotidianas, como evidencian Zhang y Capra (2019). Los docentes no solo concebían la creatividad como una creación propia, sino más bien como una motivación para que todos crearan a partir de diferentes parámetros que ellos mismos estipulaban. Algunos de ellos vieron la pandemia como una oportunidad para crear sin importar la autoría del producto. Lo anterior evidencia que a la par de docentes innovadores, se fomenta ciudadanos creativos, que muestran una solución a diferentes situaciones problémica (Sárito, 2018; Paris, 2018).

Por otra parte, los docentes que pertenecieron a la muestra evidenciaron que entre más alto su resultado del Test CREA, era mayor su organización del tiempo. y tenían estipulados sus gustos fuera del rol laboral, generando un espacio para sí mismo que los llevaba a un desahogo y tranquilidad para poder desarrollar sus competencias creativas y dar resultados a producciones divergentes, las cuales surgen de un proceso que conecta la capacidad de generar soluciones conductuales ante un imprevisto, como se evidencia en los casos (Aguilera, 2017). 


\section{Practica pedagógica}

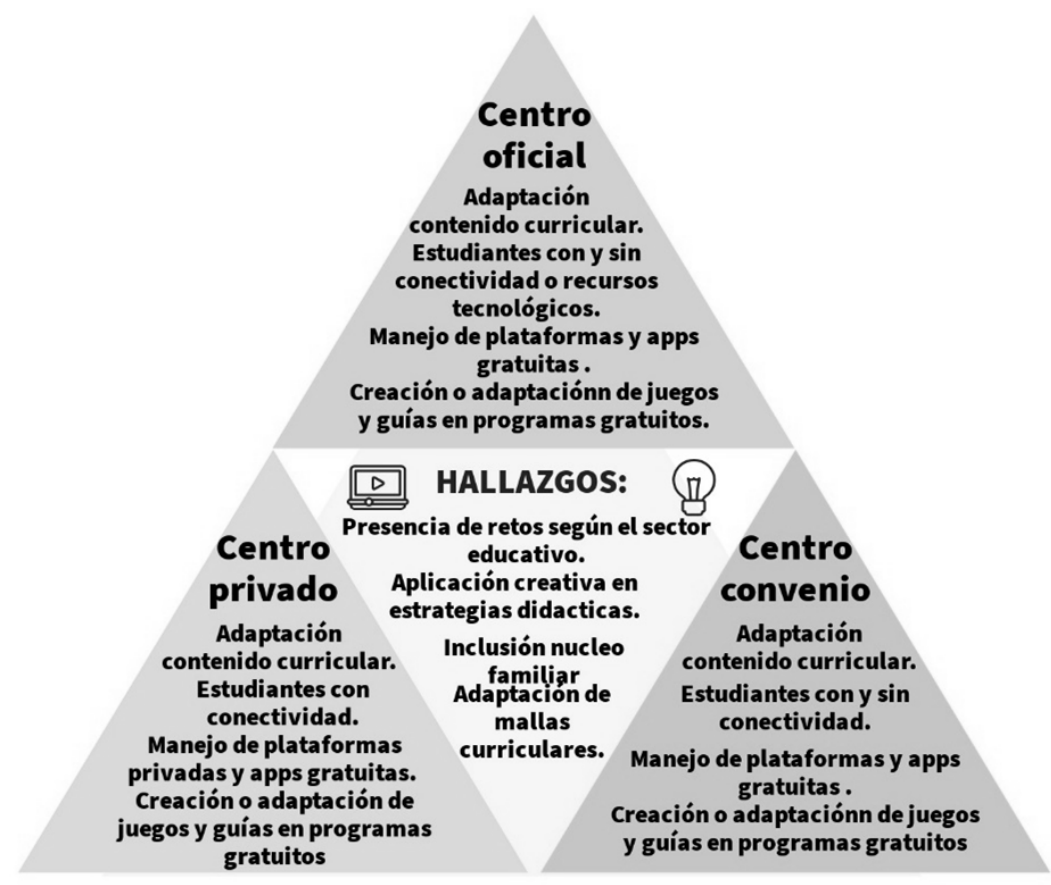

Figura 1. Triangulación de datos en la praxis pedagógica

Fuente: Elaboración propia de las investigadoras

Según la figura 10, se demostraron diferencias en la práctica pedagógica, ajustadas al nivel socioeconómico de los colegios como se muestra en la triangulación de datos en la figura 10. Según lo anterior, se manifiesta que los resultados fueron diversos, repartidos entre la conectividad, la falta de recursos económicos y la ausencia de adaptabilidad al manejo de plataformas; todo esto, producto de la situación social o económica de los padres. Según la anterior triangulación se encuentra el común denominador de la inclusión familiar al proceso cognitivo y procedimental del estudiante. Según esto, Muñoz y Lluch (2020) explican la necesidad de la familia en la educacion del año 2020, al ya no ser el docente el acompañante principal del proceso educativo, porque esté papel quedó inmerso en las responsabilidades familiares.

Por otra parte, también se evidencia un usual manejo de habilidades creativas del docente, en plataformas gratuitas, con la finalidad de producir presentaciones, videos, juegos o adaptaciones, para facilitar su saber hacer, y de esta forma mejorar la eficacia de emisión de su saber conocer (Gonzalez, 2020). A su vez, en cuanto la presencia de retos, el docente según su ubicación sectorial, tuvo que generar estrategias, demostrando que la práctica pedagógica se configura desde la experiencia, la cual requiere conocimientos que dan forma a las habilidades en el proceso de enseñanza-aprendizaje. En este sentido un 
docente es una persona de decisiones propias, que está inmerso en un proceso de adaptación, esto lo ayuda a gestionar sus habilidades de interacción y enseñanza, en presencia de diferentes dificultades (Martinez, 2017).

En este sentido, la praxis pedagógica desarrollada durante la pandemia, evidenció una clara relación con el efecto que provocó la creatividad. A su vez, se observó que los docentes, crearon y construyeron ante la falta o la limitación de recursos de conocimiento y materiales. En efecto a esto, Guillera (2020) explica la necesidad de un proceso creativo y la asociación de hábitos en los diferentes roles de la vida diaria, fundamentales para transformar o elaborar. Lo anterior explica porque los docentes con mayor índice en el Test CREA, se les facilitó superar dificultades y dominar estrategias que les permitieron flexibilizar contenidos y materiales. Como se dijo anteriormente, los docentes adaptaron estrategias, tales como videos, guías, talleres juegos y comics, y todo aquello que les facilita la carga de su labor. Por consiguiente, dentro de su saber hacer y saber conocer, tomaron la creatividad como una herramienta que les permitió sobre poner los límites y facilitar la adaptación en su saber conocer y saber hacer de su praxis (Hung \& Sitthiworachart, 2020).

\section{Discusión}

Se enfatiza en el trabajo creativo con ayuda las TIC, elaborando una dinámica constante de aprendizaje y adaptación. Esto según el teórico utilizado como antecedente, Peré (2017) demuestra una clara necesidad de formar a los docentes en nuevos conocimientos tecnológicos que promuevan la innovación del aprendizaje. A su vez según los antecedentes, basados en las investigaciones de Harris y De Bruin (2018) y Agudelo (2019), demuestra que el maestro es capaz de construir herramientas que ayudan en las dinámicas educativas. Por ser contraria, a esta idea, se discrepa con el antecedente Al-Dabobrah y Al- Zboon (2017), porque los datos analizados, demuestran que la creatividad individual no se relaciona con el sector laboral del docente, y tanto los maestros del sector privado, como el sector oficial, obtuvieron índices de inteligencia creativa alta y media.

Por otra parte, observando el objetivo de analizar la influencia creativa sobre la praxis pedagógica en tiempos de pandemia del año 2020, se analiza que esta capacidad tiene enlace con la personalidad generando una tendencia a las preferencias personales que influyen su praxis pedagógica. En efecto, esto indica que cada persona lleva un proceso diferente desarrollando conexiones distintas al realizar los procedimientos de 
respuesta, según experiencias y conocimientos (Agudelo, 2020). Según hábitos las habilidades, permiten observar una creatividad estructurada, permitiéndole construir herramientas propias que sobresalen en su praxis docente, las cuales adquieren potencial con el autoconocimiento, porque descubren habilidades que dinamizan una práctica (Garcés, 2018). Del mismo modo, la praxis pedagógica de los sujetos muestra resultados de diferentes habilidades, las cuales generan un conjunto de capacidades emocionales, personales, creativas y sociales, que producen adaptación a requerimientos y presiones en el sistema educativo (Chumbimuni, 2020). Todo lo anterior apoya los antecedentes enfocados en la idea del papel del docente como líder, y de su actitud en su saber hacer, influenciando directamente el proceso y metodología de la praxis pedagógica (Dixon \& Em, 2018; Henao \& Marin, 2019; Mul, 2019).

Por su parte, Østbye et al. (2017) en su análisis empírico basado en un modelo de ajuste regional multisectorial, demuestra que los trabajos del sector inteligente, como es la educacion, necesita personas cualificadas académica y creativamente, y se abastece de personas con estas características, las cuales cumplen con requisitos actitudinales que no se presentan en otras áreas. Lo anterior explica la razón por la cual la mayoría de los casos estudiados obtuvieron una creatividad alta. Por otra parte, en esta "actitud creativa" Campos \& Palacios, (2018) la analizan desde varios ejes de interacción única entre el sujeto, el proceso, el ambiente y el producto.

Por otro lado, el proceso creativo evidenciado, demuestra un trascurso productivo de respuesta diferente para cada uno, porque este depende de la influencia externa, la cual se enlaza con las preferencias personales (Morales, 2017). Según lo anterior, el estudio de casos arroja que la creatividad desde el paradigma personal nombrado por Barron (1981) (citado por Morales, 2017) fomenta la iniciativa desde los rasgos divergentes de pensamiento, explica la razón diferencial y variada que arrojó la muestra, refiriéndose notoriamente a las caricaturas, experimentos, adaptación de objetos de la casa y juegos creados. A su vez, tres de los casos exponían a sus estudiantes como su motivación, construyéndolos como su detonador para innovar y fomentar un acto creativo (Esquivas 2018).

\section{Conclusión}

En la investigación se analizó desde un punto fenomenológico, la incidencia de la capacidad creativa y socioemocional del docente, en su praxis pedagógica construida en la pandemia del año 2020. Esta disertación residió en el enfoque analítico de 8 casos de 
estudio, a través de una muestra diversa en colegios públicos, privados y de convenio, ubicados en Bogotá Colombia. Por lo cual llegó a examinar a través de la observación, aplicación de instrumentos y la recolección de datos, las siguientes conclusiones:

Se documentó que la creatividad del docente, referenciada por el test CREA, estuvo en la categoría alta y muy alta, mostraron flexibilidad y adaptación de herramientas, sin importar si pertenecían a la educación oficial, semioficial o privada, demostrando que la índole laboral no interfería, y aclarando que, a mayor creatividad, mayor flexibilidad. A su vez, ellos apoyaron su creatividad, adquiriendo nuevas capacidades de creación por medio de las TIC. Otro aspecto resaltante fue la relación entre el rango de creatividad y la cantidad de herramientas nuevas utilizadas durante la pandemia, pero se aclara que no todas ellas eran a través de plataformas, porque algunas se enfocaban en la utilización de materiales comunes que se encontraban en la casa, llevando a sus estudiantes a una transformación de concepto con su alrededor.

La inteligencia creativa, ayudó al docente en cuestión de generar metodologías factibles para llevaran temáticas con inventivas mixtas que incluían la presencia y ausencia de las TIC. Ahora bien, dentro de la praxis pedagógica se encontraron características empáticas, lo cual enfatizó el valor emocional a nivel propio y social, donde se resaltó el equilibrio ante una nueva realidad educativa. Lo anterior ayudó a ubicar los saberes de la praxis en un ámbito más emocional, que solventó necesidades a través de su saber hacer y saber conocer. Por otro lado, se observó que los docentes con mayor rendimiento en los test, tenían mejor manejo de tiempo útil, elaborando rutinas que abarcaban el aspecto emocional y el descanso. Por último, los docentes más creativos e inteligentes emocionalmente, se les facilitaba mantener lazos amistosos dentro y fuera de la praxis, lo cual demostraba la necesidad de tener un sistema de apoyo.

Las adaptaciones de recursos utilizados en la práctica pedagógica fueron diferentes y respondían al sector del colegio, indicando para el sector privado el uso de herramientas didácticas que explicara el manejo de diferentes plataformas, mostrando mayor grado de complejidad en la escolaridad de primaria. Por otra parte, el sector oficial respondía a necesidades económicas, como la ausencia de conectividad de los estudiantes, obligando a los docentes a utilizar metodologías de comunicación con un mínimo de ayuda en la virtualidad. Por todo lo anterior, las habilidades creativas fueron necesarias y útiles para fomentar una práctica más eficiente. 


\section{Referencias}

Arispe A., C. M.; Yangali V., J. S; Guerrero B., M.E.; Lozada R., B. O; Acuña G., L.A. \& Arellano S., C. (2020). La investigación científica. Una aproximación a los estudios de postgrado. Edit. UIDE, Guayaquil. https://repositorio.uide.edu.ec/handle/37000/4310

Agudelo, M. (2019). Habilidades creativas de los maestros del Programa Ondas Caldas. [Tesis]. Universidad Autónoma. http://repositorio.autonoma.edu.co/jspui/handle/11182/930

Aguilera, A. (2017). El pensamiento divergente: ¿qué papel juega la creatividad. Investigación y consultoria organizational. DOI: 10.6084/m9.figshare.5212429

Al-Dababneh, K. A., \& Al-Zboon, E. K. (2017). Can Teachers' Self-Reported Characteris TIC and Beliefs about Creativity Predict Their Perception of Their Creativity Practices in the Classroom. International Journal of Special Education, 32(4), 723-745. https://files.eric.ed.gov/fulltext/EJ1184189.pdf

Bernal, C. A. (2010). Metodología de la investigación. (3a ed.). Prentice Hall.

Blanquiz Y \& Villalobos M. Estrategias de Enseñanza y Creatividad del Docente en el área de Ciencias Sociales de Instituciones Educativas de Media de San Francisco. Revista Científica Arbitada. Vol. 20 Núm. 2. https://doi.org/10.36390/telos202.07

Brocki, J. M., \& Wearden, A. J. (2006). A critical evaluation of the use of interpretative phenomenological analysis (IPA) in health psychology. Psychology \& Health, 21(1), 87-108. https://doi.org/10.1080/14768320500230185

Campos G. \& Palacios A. (2018). La creatividad y sus componentes. Creatividad y Sociedad

167-183. https://www.researchgate.net/publication/323999298_La_creatividad_y_sus_co $\underline{\text { mponentes }}$

Cheung, s., Huang, E., Chang, S., \& Wei, L. (2020). Does being mindful make people more creative at work? The role of creative process engagement and perceived leader humility. Organizational Behavior and Human Decision Processes. Volume $\quad 159 . \quad$ Pages 39-48, ISSN 0749-5978. https://doi.org/10.1016/j.obhdp.2019.12.003.

Chumbimuni, K. (2019). La inteligencia emocional y habilidades sociales en docentes de secundaria de la I. E. "San Juan” de San Juan de Miraflores, 2019. [tesis]. Repositorio Institucional

UCV. http://repositorio.ucv.edu.pe/handle/20.500.12692/39143

Dacre, P., Dawne, G., \& Kath H. 2019. Developing Employable, Emotionally Intelligent, and Resilient Graduate Citizens of the Future. Employability via Higher Education: Sustainability as Scholarship, pages 83-97. DOI: 10.1007/978-3-03026342-3_6 
Déborah, M. R., \& García, M. (2018). Transformación del modelo educativo en el aprendizaje y desarrollo competencial. estudio de caso. Revista de Pedagogía, 70(4). https://doi.org/10.13042/Bordon.2018.60992

Dixon, K., \& Em, J. (2018). Teacher Emotional Intelligence and Best Practices for Classroom Management. [Tesis]. Disertación Brandman University. https://digitalcommons.brandman.edu/edd_dissertations/217

Duque, L. G., \& Duque, P. A. (2019). prácticas pedagógicas una mirada a la formación profesional de enfermería - pedagogical practices: a look to the nursing professional education. Revista de Investigaciones. https://doi.org/10.22383/ri.v19i33.127

Eden, D. (2020). The Resilience of Maintained Education in England in the Face of a Worldwide Pandemic. Forum: For Promoting 3-19 Comprehensive Education, 62(3), 323-334. https://doi.org/10.15730/forum.2020.62.3.323

Elisondo, R. (2018) Creatividad y educación: llegar con una buena idea; Asociación para la Creatividad. Creatividad y sociedad; 27; 12-2018; 145-166 https://ri.conicet.gov.ar/handle/11336/98611

Extremera, N., Mérida-López, S., Sánchez-Álvarez, N., \& Quintana-Orts, C. (2018) How Does Emotional Intelligence Make One Feel Better at Work? The Mediational Role of Work Engagement. Int. J. Environ Public Health 2018, 15, 1909. https://www.mdpi.com/1660-4601/15/9/1909\#cite

Flores, C. A. (2019). Inteligencia emocional en docentes de una Institución Educativa Pública del distrito mí Perú-Callao. [tesis]. Universidad San Ignacio de Loyola http://repositorio.usil.edu.pe/handle/USIL/8802

Garcês, S. (2018). Creativity in science domains: A reflection. Atenea, 517, 241-253. http://revistasacademicas.udec.cl/index.php/atenea/article/view/544

Garcês, S. (2018). Creativity in science domains: A reflection. Atenea, 517, 241-253. http://revistasacademicas.udec.cl/index.php/atenea/article/view/544

Gonzalez Y. (2020, 16 de septiembre). La educacion pospandemia. Opinión semanario universidad.

Semanario

Universidad. https://semanariouniversidad.com/opinion/la-educacion-pospandemia

Guilera Ll (2020). Anatomía de la creatividad. Marge Books. ISBN 978-84-17903-54-1. Recuperado de: https://books.google.es/books?hl=es\&lr\&id=7MbpDwAAQBAJ\&oi=fnd\&pg=P A11\&dq=PERSONAS+CREATIVAS\&ots=1QFa0gphi3\&sig=zaAFjW3jgsZyY xtOPHZNcvP_r84\&pli=1\#v=onepage\&q=PERSONAS\%20CREATIVAS\&f=fal se

Gustafsson, M., \& Nuga, D. (2020). How is the COVID-19 pandemic affecting educational quality in South Africa? Evidence to date and future risks. Working Papers from Stellenbosch University. 
Hacımustafaoğlu M. (2020). COVID-19 and re-opening of schools: Opinions with scientific evidence. Turk Pediatri Arsivi 2020; 55(4). https://www.ncbi.nlm.nih.gov/pmc/articles/PMC7750348/

Haertel T \&Terkowsky C. (2021) Online Creativity in Engineering Education. In: Auer M., May D. (eds) Cross Reality and Data Science in Engineering. REV 2020. Advances in Intelligent Systems and Computing, vol 1231. Springer, Cham. https://doi.org/10.1007/978-3-030-52575-0_43

Hamed,J., \& Ahmad, S. (2019) Beliefs about Scientific Creativity Held by Pre-Service Science Teachers in the State of Kuwait. Canadian Center of Science and Education. https://eric.ed.gov/?id=EJ1230559

Harris, A., y De Bruin, L. R. (2018). Secondary school creativity, teacher practice and STEAM education: An international study. Journal of Educational Change, 19(2), 153-179. https://doi.org/10.1007/s10833-017-9311-2

Henao, J. F., y Marín, A, E. (2019). El proceso de enseñanza desde el prisma de las emociones de los docentes. Praxis \& Saber, 10(24), 193-215. https://revistas.uptc.edu.co/index.php/praxis_saber/article/view/9415

Hernández-Sampieri, R. \& Mendoza, C (2018). Metodología de la investigación. Las rutas cuantitativa, cualitativa y mixta, Ciudad de México, México: Editorial Mc Graw Hill Education, Año de edición: 2018, ISBN: 978-1-4562-6096-5, 714 p.

Hernández-Sampieri, R. \& Mendoza, C (201b). Metodología de la investigación, cartilla adicional. Las rutas cuantitativa, cualitativa y mixta, Ciudad de México, México: Editorial Mc Graw Hill Education, Año de edición: 2018, ISBN: 978-1-45626096-5, 714 p.

Hung, W., \& Sitthiworachart, J. (2020) J. In-Service Teachers' Conception of Creativity and Its Relation with Technology: A Perspective from Thailand. The Asia-Pacific Education Researcher. https://doi.org/10.1007/s40299-019-00460-6

Kotsou, I., Mikolajczak, M., Heeren, A., Grégoire, J., \& Leys, C. (2019). Improving Emotional Intelligence: A Systematic Review of Existing Work and Future Challenges. Emotion Review, 11(2), 151165. https://doi.org/10.1177/1754073917735902

Mays, T. (2020). Towards More Resilient Schooling: Possible Models for the Future. En Commonwealth of Learning. Commonwealth of Learning

Markovits, E., \& Douglas, A. (2020). Pandemic Innovation. New England Journal of Higher

Education.

https://eric.ed.gov/?q=pandemic+and+education\&id=EJ1265814

Martinez, M. (2017, mayo 15). Saber pedagógico, práctica pedagógica y formación docente. Magisterio, 83. https://www.magisterio.com.co/articulo/saberpedagogico-practica-pedagogica-y-formacion-docente 
Mattingly, V., \& Kurt, K. (2019). Can emotional intelligence be trained? A metaanalytical investigation. Human Resource Management Review, Volume 29, Issue 2, 2019. https://doi.org/10.1016/j.hrmr.2018.03.002.

Miranda, F. (2020, Julio 23,). Regreso a clases presenciales. Revista Portafolio. https://www.portafolio.co/opinion/francisco-miranda-hamburger/regreso-aclases-presenciales-542981

Morales, C. (2017). La creatividad, una revisión científica. Revista científica de arquitectura. https://rau.cujae.edu.cu/index.php/revistaau/article/view/420

Mull, J. C. (2019). Hiding in Plain Sight: Exploring Middle School Teachers' Accounts of “Creativity for Teaching”. En ProQuest LLC. ProQuest LLC. [ tesis]. Universidad de

Pennsylvania. https://repository.upenn.edu/dissertations/AAI13859102/

Muñoz, J., \& Lluch, L. (2020). Educación y Covid-19: Colaboración de las Familias y Tareas escolares. Revista Internacional de Educación para la Justicia Social, 9(3), 1-17, e-ISSN: 2254-3139. https://revistas.uam.es/riejs/article/view/12182

Østbye, S., Moilanen, M., Tervo, H., \& Westerlund, O. (2018). The creative class: Do jobs follow people or do people follow jobs? Regional Studies, 52(6), 745-755. https://doi.org/10.1080/00343404.2016.1254765

Perasso, V. (2016, octubre 12). Qué es la cuarta revolución industrial (y por qué debería preocuparnos). BBC News Mundo. https://www.bbc.com/mundo/noticias37631834

Peré (2017). Apuntes para analizar la relación entre innovación, TIC y formación pedagógico-didáctica. Praxis \& Saber, 8(16), 15-33. https://doi.org/10.19053/22160159.v8.n16.2017.6165

Rico, A. (2017). Evaluación del uso de las APPs que abordan los procesos creativos en la educación artística formal. https://doi.org/10.35376/10324/22663

Sárito, A (2018). Personas creativas ciudadanos creativos. ISSN 978-958-763-326-9. Corporación Universitaria Minuto de Dioshttps://hdl.handle.net/10656/7364

Snyder H., Sowden P., Silva P., \& Laufman J (2019). The creative self: Do people distinguish creative self-perceptions, efficacy, and personal identity? Psychology of Aesthe TIC, Creativity and the Arts (original publicado en 2012). https://core.ac.uk/download/pdf/288919386.pdf

Vesely-Maillefer A.K., \& Saklofske D.H. (2018) Emotional Intelligence and the Next Generation of Teachers. In: Keefer K., Parker J., Saklofske D. (eds) Emotional Intelligence in Education. The Springer Series on Human Exceptionality. Springer, Cham. https://doi.org/10.1007/978-3-319-90633-1_14

Wajdi, M., Rahayu, S., Ulfatin, N., Wiyono, B., \& Imron, A . (2018). The Professional Competency Teachers Mediate the Influence of Teacher Innovation and Emotional Intelligence on School Security. Journal of Social Studies Education 
$\begin{array}{lllll}\text { Research, } & 9 & \text { (2), } & \text { 210-227. } & \text { Retrieved }\end{array}$ https://dergipark.org.tr/en/pub/jsser/issue/37944/438292

Zhang, Y., \& Capra, R. Understanding How People use Search to Support their Everyday Creative Tasks. Proceedings of the 2019 Conference on Human Information Interaction and Retrieval. March 2019 Pages 153-162. https://doi.org/10.1145/3295750.3298936 